\title{
EXAFS STUDIES ON STRUCTURAL DISORDER IN CALCIUM DEFICIENT HYDROXYAPATITE
}

\author{
Masato Tamai, ${ }^{1}$ and Atsushi Nakahira ${ }^{1 *}$ \\ (*e-mail; nakahira@kit.ac.jp) \\ ${ }^{1}$ Department of Chemistry and Materials Technology, Kyoto Institute of Technology,Matsugasaki, Sakyo-ku, \\ Kyoto, 606-8585, Japan
}

Keywords: Hydroxyapatite, hydrothermal treatment, $\mathrm{Ca} / \mathrm{P}$ molar ratio, $\mathrm{Ca}$ vacancy, EXAFS spectroscopy

\begin{abstract}
The structural disorder in calcium-deficient hydroxyapatite (Ca-def HAp) was investigated by extended X-ray absorption fine structure spectroscopy (EXAFS) and X-ray absorption near edge structure spectroscopy (XANES). $\mathrm{Ca}$-def HAp was prepared by hydrolysis of $\alpha$-tricalcium phosphate. The $\mathrm{Ca} / \mathrm{P}$ molar ratio in Ca-def $\mathrm{HAp}$ was 1.54 . In order to synthesize Ca-def $\mathrm{HAp}$ with various $\mathrm{Ca} / \mathrm{P}$ molar ratios, a hydrothermal treatment in $\mathrm{Ca}\left(\mathrm{NO}_{3}\right)_{2}$ aqueous solution for Ca-def HAp was carried out. The $\mathrm{Ca} / \mathrm{P}$ molar ratio in the sample obtained by the hydrothermal treatment increased linearly with increasing reaction time. By hydrothermal treatment for 13 days, the $\mathrm{Ca} / \mathrm{P}$ molar ration in the sample increased up to 1.65 which almost stoichiometric ratio. Ultraviolet-Visible (UV-VIS) absorption at 280nm appeared in Ca-def HAp and the peak intensities decreased with increasing $\mathrm{Ca} / \mathrm{P}$ molar ratio in Ca-def HAps. The UV-VIS absorption, therefore, could be identified as intrinsic peak due to $\mathrm{Ca}$ vacancies in the HAp lattice. The fact also would suggest that $\mathrm{Ca}$ ions in the solution were taken into the vacancies in the lattice during the hydrothermal treatments. Intensity of background subtracted and normalized XANES spectra of Ca-def HAp was weaker than that of stoichiometric HAp The reason would be why the distortions are introduced by loosing of structure resulting from deficiency of $\mathrm{Ca}$ ion in HAp lattice. From EXAFS analysis of both Ca-def HAp and stoichiometric HAp, it was revealed that the coordination number of $\mathrm{Ca}$ ion in $\mathrm{Ca}$-def $\mathrm{HAp}$ was lower than stoichiometric HAp. In addition, the Ca-O distance in Ca-def HAp became to be slightly longer than that of stoichiometric HAp Therefore, it could be concluded that the structural disorder resulting from deficiency of $\mathrm{Ca}$ ions will caused the extension of Ca-O distance in HAp structure.
\end{abstract}

(Received July 5, 2004; Accepted August 25, 2004)

\section{INTRODUCTION}

Calcium-deficient hydroxyapatite (Ca-def HAp, $\left.\mathrm{Ca}_{10-\mathrm{Z}}\left(\mathrm{HPO}_{4}\right)_{\mathrm{Z}}\left(\mathrm{PO}_{4}\right)_{6-\mathrm{Z}}(\mathrm{OH})_{2-\mathrm{Z}}, \mathrm{Z}=0-1\right)$, which has lower $\mathrm{Ca} / \mathrm{P}$ ratio than that of stoichiometric $\mathrm{HAp}$ (s-HAp, $\mathrm{Ca}_{10}\left(\mathrm{PO}_{4}\right)_{6}(\mathrm{OH})_{2}$, hexagonal, $\mathrm{a}=0.943 \mathrm{~nm}$, $\mathrm{c}=0.688 \mathrm{~nm}$, space group ; $\left.\mathrm{P}_{3} / \mathrm{m}\right)$, is of greater biological interest than $\mathrm{s}-\mathrm{HAp}$ since the $\mathrm{Ca} / \mathrm{P}$ molar ratio in bone is lower than 1.67. It has been suggested that Ca-def HAp plays important roles in several processes such as bone remodeling and bone formation $^{1,2}$. Various studies about Ca-def HAp have been carried out ${ }^{3,4,5,6,7}$. However, detailed structural information for Ca-def HAp is not enough, since structural change involving $\mathrm{Ca}$ ions deficiency from the HAp lattice is too slight to analyze, accurately.

Extended X-ray absorption fine structure (EXAFS) is the variation in the absorption coefficient of an element on the high-energy side of the $\mathrm{X}$-ray absorption edge $\mathrm{e}^{8,9}$. The variation is a result of the interference between a primary photoelectron wave emitted by an atom, on absorption of an X-ray photon and secondary back scattered from neighboring atom. The interwave depends on the precise geometry of the atomic environment around the emitting atom. Consequently, EXAFS can provide information on the coordination distance of atoms from the excited atom. Therefore, EXAFS is one of most useful tool for structural analysis. Harries and co-workers have investigated the EXAFS spectra of $\mathrm{HAp}^{10}$ and shown qualitative difference in the structural environment around calcium in carbonated apatite ${ }^{11}$.

The purpose of this study is to investigate the structural difference in s-HAp and Ca-def HAp. Especially, from EXAFS spectra of several Ca-def $\mathrm{HAps}$ with various $\mathrm{Ca} / \mathrm{P}$ molar ratios, structural disorder resulting from deficiency of $\mathrm{Ca}$ ion is discussed in the present paper.

\section{MATERIALS AND METHODS}

Synthesis of Ca-def HAps with various the Ca/P molar ratio 
Tamai M, Nakahira A

Ca-def HAp was prepared by hydrolysis of $\alpha$-tricalcium phosphate ( $\alpha$-TCP Taihei Chemical Industries, co., ltd.). $\quad 0.01 \mathrm{~mol}$ of $\alpha$-TCP was stirred for $48 \mathrm{~h}$ at $70^{\circ} \mathrm{C}$ in 1 -octanol $/ \mathrm{H}_{2} \mathrm{O}$ binary solvent $(100 \mathrm{ml} / 60 \mathrm{ml})$. The details for the synthesis of the Ca-def HAp refer to our previous paper $^{12}$.

Controlling of the $\mathrm{Ca} / \mathrm{P}$ molar in Ca-def HAp were carried out by hydrothermal treatment. At first, $0.5 \mathrm{mmol}$ of Ca-def HAp and $50 \mathrm{ml}$ of $0.5 \mathrm{~mol} / 1-\mathrm{Ca}\left(\mathrm{NO}_{3}\right)_{2}$ were mixed. The $\mathrm{pH}$ value of the suspension was adjusted to 10 with ammonia aqueous solution. Next, the suspension was placed in double-walled vessel, which the inner vessel was made of Teflon and the outer vessel was made of stainless steel. The vessel was introduced into a drying oven that was preheated at $150^{\circ} \mathrm{C}$ and it was held 1-13days.

\section{Characterizatios}

The obtained samples were identified by powder X-ray diffraction analysis (XRD, RINT $2500 /$ Rigaku co.,ltd) using $\mathrm{CuK}_{\alpha}$ radiation $(40 \mathrm{kV}, 50 \mathrm{~mA})$ The XRD profiles were collected between $20-60^{\circ}$ of $2 \theta$ angles with a step interval of $0.01^{\circ}$ and scanning rate of $4^{\circ} / \mathrm{min}$. The atomic concentrations of calcium and phosphorus in Ca-def HAp were performed by X-ray fluorescence spectrometer (XRF, XRF-1700 / Shimadzu co., ltd.) using $\mathrm{Rh} \mathrm{K}_{\alpha}$ radiation $(40 \mathrm{kV}, 95 \mathrm{~mA})$. The microstructural evaluation was carried out by transmission electron microscopy (TEM, JEM-2010SP /JEOL) with accelerating voltage of $200 \mathrm{kV}$.

Several Ca-def HAps were also identified by infrared absorption spectroscopy (IR: IR-WINSPEC100 / JEOL) and ultraviolet-visible absorption spectroscopy (UV-VIS, UV2400 / Shimazu co., ltd.) The Ca K-edge X-ray absorption near edge structure (XANES) and extended X-ray absorption fine structure (EXAFS) spectra were recorded at room temperature at BL01B1 in SPring8, Japan synchrotron radiation facility. The operating conditions in the storage ring were the following: positions at energies of $8.00 \mathrm{GeV}$ and intensities of about 150 $\mathrm{mA}$. The Ca-K edge XANES and EXAFS data for the study was corrected by transmission mode using the two-crystal Si (111) monochromator. The photon flux was measured by two ionization chambers flushed by a $\mathbf{H e}: \mathbf{N}_{2}=\mathbf{7 0} \%: 30 \%$ mixture gas (I0) and $\mathrm{N}_{\mathbf{2}}: \mathbf{1 0 0 \%}$ (I). Ti metallic foil was used to check the energy calibration. The energy was scanned by $0.25 \mathrm{eV}$ steps over the energy range from 4020 to $4120 \mathrm{eV}$ for the $\mathrm{Ca}$ $\mathrm{K}$-edge XANES, while the energy was scanned by
$2.00 \mathrm{eV}$ steps over the energy range from 4850 to $5600 \mathrm{eV}$ for EXAFS. XANES were processed by subtracting a linear background computed by least-square fitting from the preedge region and normalized by using the beginning of EXAFS oscillation as a unit. EXAFS modulations were analyzed by using standard methods ${ }^{13,14,15}$. The continuous absorption background was estimated by fitting the spectrum before the edge by a Victoreen function. Structural information was extracted by single-scattering theory using software made by Rigaku (REX2000). The $k^{3}$-weighed EXAFS spectra were obtained from normalized EXAFS spectra and Fourier transformations were performed within the range of $\Delta k=4-15 \AA^{-1}$ without any phase shift correction. Curve-fitting analysis were performed within $\Delta k=2.225-12 \AA^{-1}$ with the empirical parameter extracted from well-crystallized $\mathrm{CaO}$ for $\mathrm{Ca}-\mathrm{O}$ shell in the range of $\Delta \mathrm{R}=1.4-2.3 \AA$

\section{RESULTS AND DISCUSSION}

\section{Synthesis of Ca-def HAps with various the Ca/P molar ratio}

XRD profiles of sample prepared by hydrolysis of $\alpha$-TCP were composed by several peaks only due to crystal structure of hydroxyapatite. Also, the $\mathrm{Ca} / \mathrm{P}$ molar ratio in the sample was 1.54 . The sample, therefore, would be identified as Ca-def HAp. On the other hand, XRD patterns of the samples obtained by hydrothermal treatment of the Ca-def $\mathrm{HAp}$ in $\mathrm{Ca}\left(\mathrm{NO}_{3}\right)_{2}$ solution show monolithic hydroxyapatite phase. TEM images of the samples obtained by hydrothermal treatment in $\mathrm{Ca}\left(\mathrm{NO}_{3}\right)_{2}$ solution for various reaction time are shown in Fig.1. The sample obtained by hydrolysis of $\alpha$-TCP has a whisker-like morphology elongated along c-axis with $2-5 \mu \mathrm{m}$ in length and $0.1 \mu \mathrm{m}$ in diameter (Fig1(a)). On the other hand, in case of the sample obtained by hydrothermal treatment for 3 days (b), 7days(c) and 13 days(d), the microstructure is almost the same as that of before hydrothermal treatment.

The $\mathrm{Ca} / \mathrm{P}$ molar ratio in the sample prepared by hydrothermal treatment is shown in Fig.2. The $\mathrm{Ca} / \mathrm{P}$ molar ratio in Ca-def $\mathrm{HAp}$ increases linearly with an increase reaction time. By hydrothermal treatment for 13 days, the $\mathrm{Ca} / \mathrm{P}$ molar ration in the sample increased up to 1.65 which almost stoichiometric ratio (the sample named as s-HAp in the present study). Fig.3 shows UV-VIS spectra of Ca-def HAp with various $\mathrm{Ca} / \mathrm{P}$ molar ratios. As shown in Fig.3, in case of Ca-def HAp with the $\mathrm{Ca} / \mathrm{P}$ molar ratio of 1.54 , the 

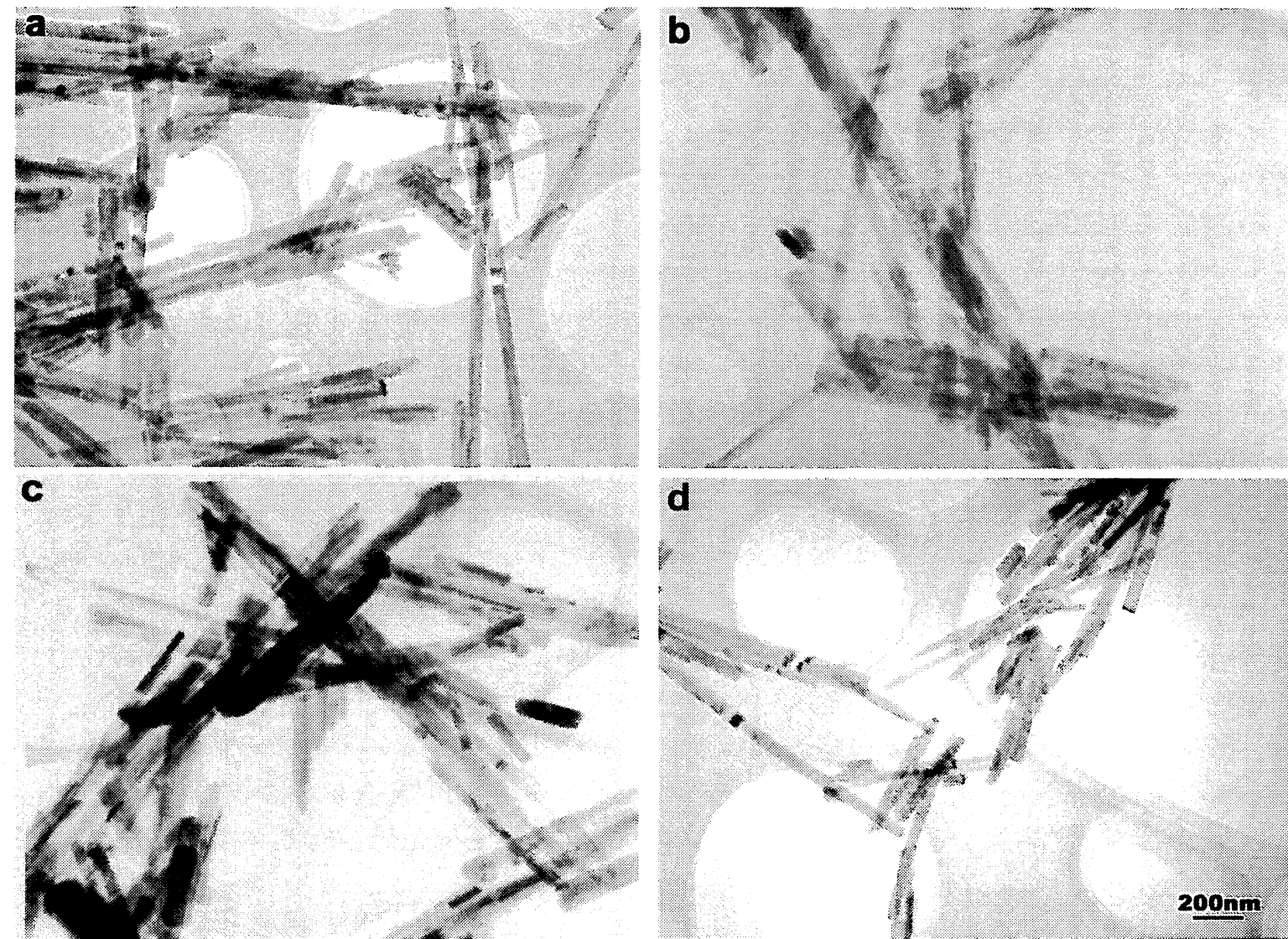

Fig.1. TEM images of the samples obtained by hydrothermal treatment in $\mathrm{Ca}\left(\mathrm{NO}_{3}\right)_{2}$ solution. (a) before hydrothermal treatment, (b) for 3days, (c) for 7days and (d) for 13days

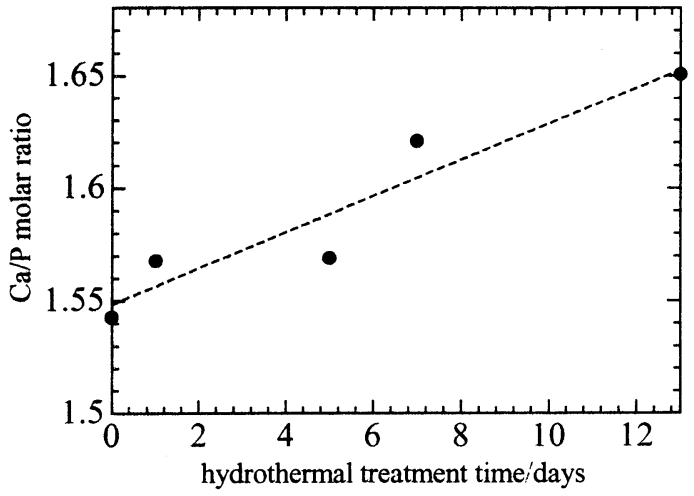

Fig.2. $\mathrm{Ca} / \mathrm{P}$ molar ratio in $\mathrm{Ca}$-def $\mathrm{HAp}$ by hydrothermal treatment.

broad UV absorption from 240-350nm, which peak top position is at about $280 \mathrm{~nm}$, is observed. The intensities of absorption peaks increase with a decrease $\mathrm{Ca} / \mathrm{P}$ molar ratio in Ca-def HAps. Finally, the absorption peak became to be weakening. As well known as a color center, intrinsic energy levels are formed in forbidden band in the crystals with vacancies. Therefore, the absorption peak at about $280 \mathrm{~nm}$ would be due to

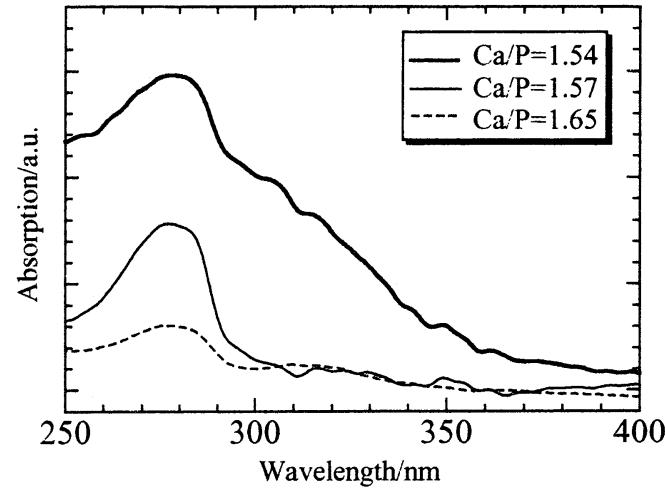

Fig.3. UV-VIS spectra of Ca-def HAp with various $\mathrm{Ca} / \mathrm{P}$ molar ratios.

band absorption resulting from $\mathrm{Ca}$-vacancies in Ca-def HAp. In addition, results of $\mathrm{Ca} / \mathrm{P}$ molar ratio and UV-VIS spectra would suggest that $\mathrm{Ca}$ ions in the solution were taken into the vacancies in the lattice during the hydrothermal treatments. As described above, Ca-def HAp can be regarded as non-stoichiometric HAp with Ca-vacancies. In the present study, the structural disorder resulting 


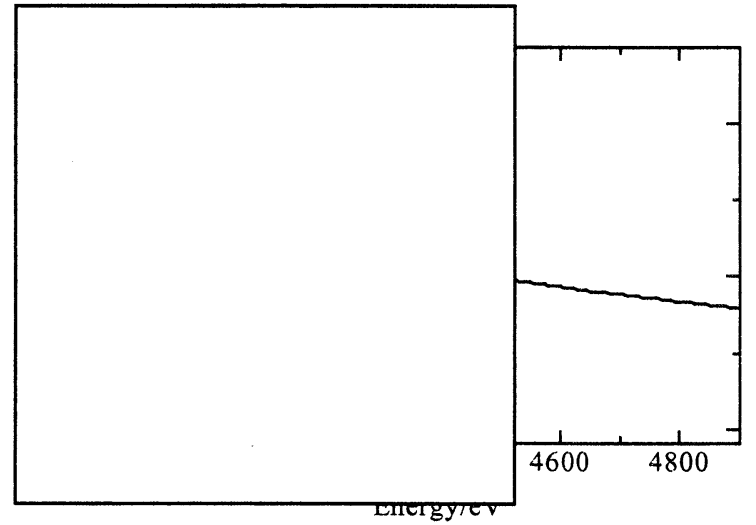

Fig.4. XAFS spectra of $\mathrm{s}-\mathrm{HAp}(\mathrm{Ca} / \mathrm{P}=1.65)$

from $\mathrm{Ca}$ vacancies were investigated by EXAFS spectroscopy.

Ca-K edge XAFS (XANES and EXAFS) of stoichiometric HAp and Ca-def HAp

In XAFS spectra, the quality of spectra is so important, since the accuracy of quantitative analysis are strongly depended on the quality of the spectra. Fig.4 shows XAFS spectra of stoichometric hydroxyapatite. As shown in Fig.4, the obtained XAFS spectra in the present study show good quality.

Background subtracted and normalized $\mathrm{Ca}$ K-edge XANES spectra of s-HAp obtained by hydrothermal treatment for 13 days (the $\mathrm{Ca} / \mathrm{P}$ molar ratio is 1.65 ) and Ca-def HAp HAp with $\mathrm{Ca} / \mathrm{P}$ molar ratio of 1.54 are shown in Fig.5. It is confirmed that the peak intensity of XANES spectra in Ca-def HAp are weaker than that in $\mathrm{s}-\mathrm{HAp}$ The main absorption are resulting from an electron transition from $1 \mathrm{~s}$ to $3 p$ and spectra reflect to the structure and/or symmetry of the compound. Since there have been only a few studies discussing HAp structure using Ca-K edge XANES, the consensus on an interpretation for $\mathrm{Ca} \mathrm{K}$ edge XANES is not established. However, for the peak intensity of K edge XANES, Minaki ${ }^{16}$ reported that, in case of structural disorder was introduced by any reasons, broadening occurred in unocuupied $3 p$ orbital and then an intensity of X-ray absorption due to electron transition $1 \mathrm{~s}$ to $3 \mathrm{p}$ will decrease. According to this report, it is considered that the reason that decreases of intensity in XANES spectra is why the distortions are introduced by deficiency of $\mathrm{Ca}$ ion in HAp lattice in Ca-def HAp.

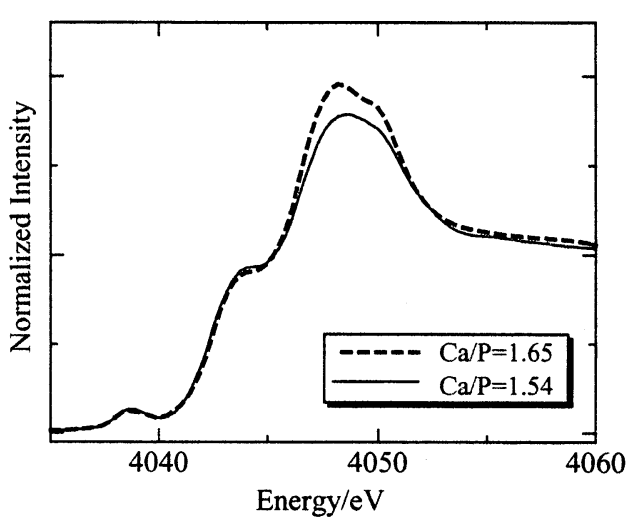

Fig.5 Ca K-edge XANES spectra of s-HAp $(\mathrm{Ca} / \mathrm{P}=1.65)$ and $\mathrm{Ca}$-def $\mathrm{HAp}(\mathrm{Ca} / \mathrm{P}=1.54)$

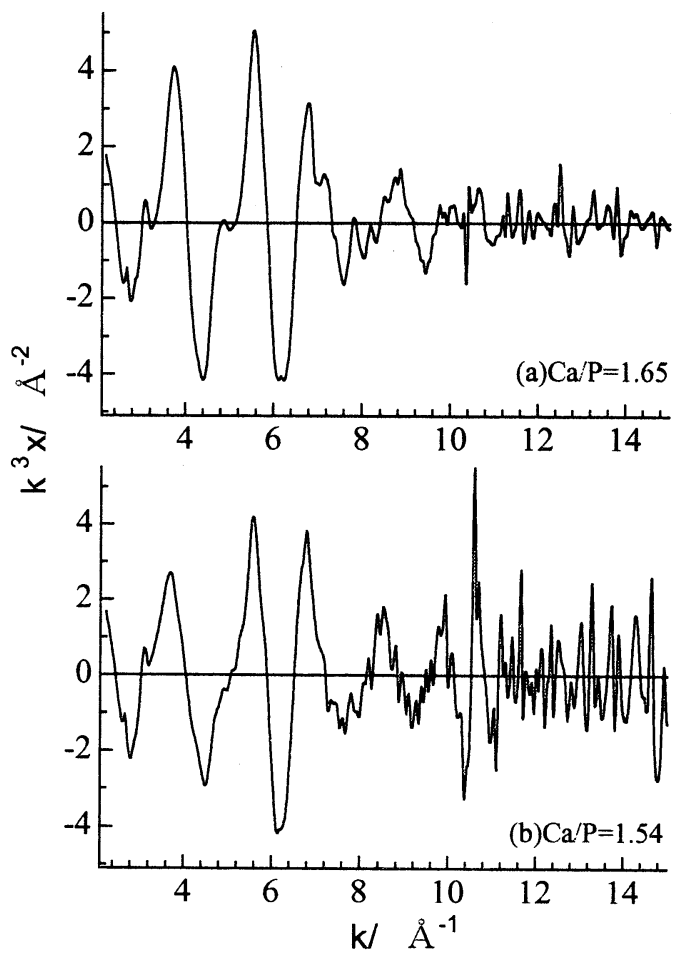

Fig.6. $\mathrm{Ca}$ K-edge EXAFS oscillations of (a)s-HAp $(\mathrm{Ca} / \mathrm{P}=1.65)$ and (b) Ca-def $\operatorname{HAp}(\mathrm{Ca} / \mathrm{P}=1.54)$ 


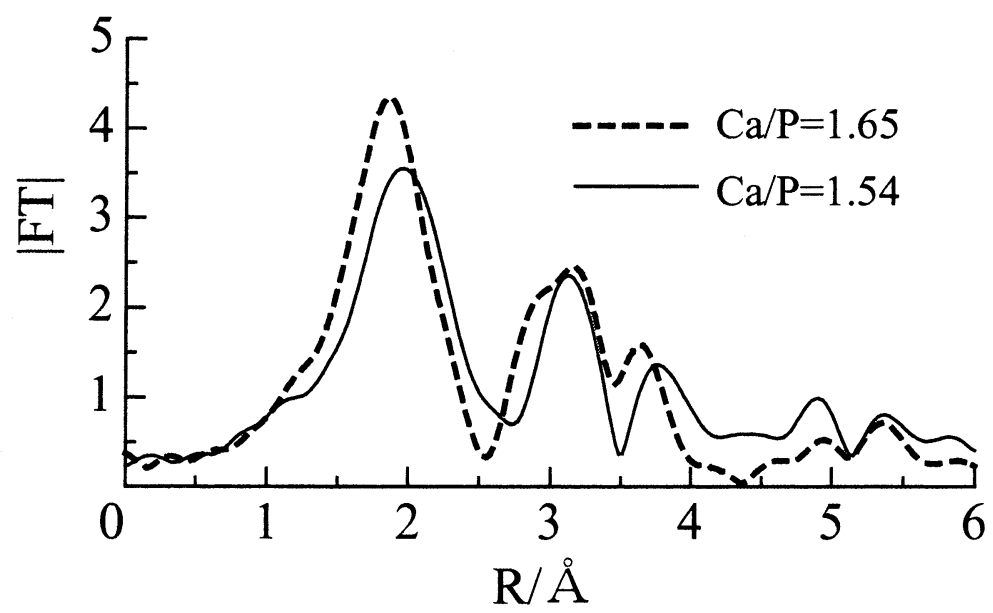

Fig.7. FT-EXAFS of Ca-def $\mathrm{HAp}(\mathrm{Ca} / \mathrm{P}=1.54)$ and $\mathrm{s}-\mathrm{HAp}(\mathrm{Ca} / \mathrm{P}=1.65)$
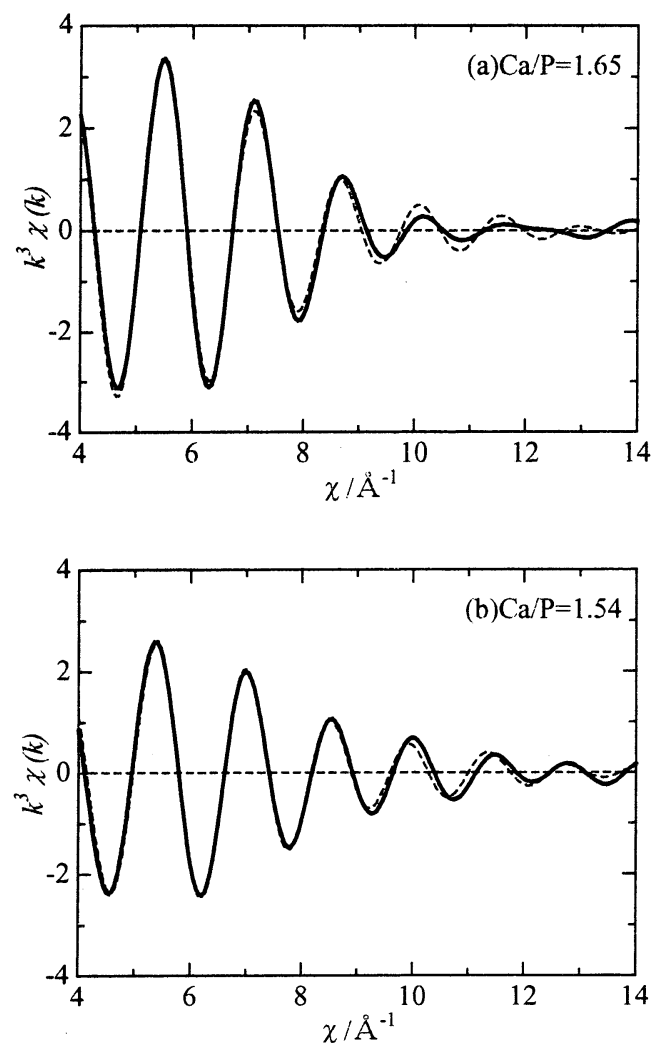

Fig.8. The best fitting of back-filtered EXAFS oscillations in the range 1.4-2.3 $\AA$. Bold lines are the isolated EXAFS and dotted lines are the model EXAFS obtained by one shell fitting of $\mathrm{Ca}-\mathrm{O}$.
Table1. Results of EXAFS analysis by curve-fitting $\left(\Delta k=2.225-12 \AA^{-1}, \quad \Delta \mathrm{R}=1.4-2.3 \AA\right)$

\begin{tabular}{ccccc}
\hline Sample & $\mathrm{N}$ & $\mathrm{R} / \AA$ & $\mathrm{dE}(\mathrm{eV})$ & $\mathrm{R}$ factor \\
\hline $\mathrm{Ca} / \mathrm{P}=1.65$ & 8.2 & 2.429 & 2.614 & 1.288 \\
$\mathrm{Ca} / \mathrm{P}=1.54$ & 7.1 & 2.467 & 2.033 & 1.861 \\
\hline
\end{tabular}

EXAFS analysis was carried out to obtain the structural parameters of $\mathrm{Ca}$ ions in HAp. $k^{3}$-weighted EXAFS oscillations of Ca-def HAp(a) and s- HAp(b) are shown in Fig.6. The oscillation waves of both s-HAp and Ca-def HAp consist of almost a single sine wave. The EXAFS oscillation of Ca-def HAp is slightly different from that of s-HAp in the amplitude. Fig.7 shows the Fourier transformed (FT) EXAFS of both s-HAp and Ca-def HAp. Considering to atomic arrangement of HAp, the first FT peak can be identified as $\mathrm{Ca}-\mathrm{O}$ scattering. Notably, the first peak in Ca-def HAp slightly shifted to long distance side comparing to that of s-HAp To obtain a detailed local structure, the curve fitting of the $\mathrm{Ca}-\mathrm{O}$ shell within $\Delta \mathrm{R}=1.4-2.3 \AA$ was performed by inverse FT after FT-filtered EXAFS in the fitting range of $\Delta k=2.225-12 \AA^{-1}$. In Fig.6, the best fits for s-HAp and Ca-def HAp are shown. In both samples one shell fitting of the $\mathrm{Ca}-\mathrm{O}$ shell 


\section{Tamai M, Nakahira A}

gave satisfactory results. The parameters obtained by the EXAFS analysis of s-HAp and Ca-def HAp are summarized in Tablel. Average coordination number of s-HAp is about 8.2, while in case of Ca-def HAp is about 7.1. $\mathrm{Ca}$ ions in s-HAp occupy two non-equivalent positions. $\mathrm{Ca}(\mathrm{I})$ ions are on the threefold axis at $1 / 3,2 / 3, \mathrm{Z}$ (where $\mathrm{z}$ is usually $<0.01$ ) and three other equivalent site. $\mathrm{Ca}$ (II) ions are on the miorr plane at 0.01 , $0.26,1 / 4$ and five other equivalent site. $\quad \mathrm{Ca}(\mathrm{I})$ ions are coordinated by nine oxygen atom from six phosphate ions and $\mathrm{Ca}$ (II) ions are coordinated by six oxygen atom from five phosphate ions and one oxygen atom from hydroxyl ions. Since the coordination number calculated from curve fitting is average value, the coordination number of s-HAp will be appropriate value. As discussed above, Ca-def HAp can be regarded as non-stoichiometric apatite with $\mathrm{Ca}$ vacancies. Therefore, it is considered that the coordination number of Ca-def HAp will decrease resulting from deficiency of $\mathrm{Ca}$ ion in the lattice. On the other hand, $\mathrm{Ca}-\mathrm{O}$ distance of s-HAp and $\mathrm{Ca}-\mathrm{def}$ HAp with $\mathrm{Ca} / \mathrm{P}$ molar ratio of 1.54 is $2.429 \AA$ and $2.467 \AA$, respectively. The $\mathrm{Ca}-\mathrm{O}$ distance in Ca-def HAp became to be longer than that of s-HAp Therefore, it could be concluded that the structural disorder resulting from deficiency of $\mathrm{Ca}$ ions will caused the extension of $\mathrm{Ca}-\mathrm{O}$ distance in $\mathrm{HAp}$ structure.

\section{CONCUSION}

The structural disorder in Ca-def HAp was investigated by mainly XANES and EXAFS spectroscopy. Those findings in the present study are summarized as following.

(1) The $\mathrm{Ca} / \mathrm{P}$ molar ratio in the sample obtained by the hydrothermal treatment increased linearly with increasing reaction time. By hydrothermal treatment for 13 days, the $\mathrm{Ca} / \mathrm{P}$ molar ration in the sample increased up to 1.65

(2) UV-VIS absorption at 290nm appeared in $\mathrm{Ca}$-def HAp and the peak intensities decreased with increasing $\mathrm{Ca} / \mathrm{P}$ molar ratio in $\mathrm{Ca}$-def $\mathrm{HAp}$. The UV-VIS absorption could be identified as intrinsic peak due to $\mathrm{Ca}$ vacancies in the HAp lattice.

(3) Intensity of background subtracted and normalized XANES spectra of Ca-def HAp was weaker than that of stoichiometric HAp The reason would be why the distortions are introduced by loosing of structure resulting from deficiency of Ca ion in HAp lattice.
(4) From EXAFS analysis of both Ca-def HAp and s-HAp, it was revealed that the coordination number of $\mathrm{Ca}$ ion in Ca-def HAp was lower than stoichiometric HAp and the $\mathrm{Ca}-\mathrm{O}$ distance in Ca-def HAp became to be slightly longer than that of stoichiometric HAp Therefore, the structural disorder resulting from $\mathrm{Ca}$ vacancies would caused the extension of $\mathrm{Ca}-\mathrm{O}$ distance in HAp structure.

\section{ACKNOWLEDGEMENTS}

X-ray absorption experiment was carried out under the approval of JASRI (proposal No. 2001A0227-NX-np). We thank Dr. T.Uruga at JASRI for his help.

\section{REFERENCES}

1 L.L.Hench, J. Am. Ceram. Soc. 74 1487(1991)

2 A.S.Posner, Physiol. Rev.49 760(1969)

3 H.Monma, S. Ueno, Y. Tsutsumi and K. Kanazawa, J.Ceram.Soc.Jpn.(Yogyo-Kyokai-shi) 86 590(1978)

4 T.Kanazawa in "Inorganic Phosphate Chemistry", (Kodansha scientific Tokyo ,1985)

5 I. R. Gibson, I. Rehaman, S. M. Best and W. Bonifild, J. Mater.Sci. in Med. 12799 (2000)

6 M.Tamai, T.Isshiki, K.Nishio, M.Nakamura, A.Nakahira and H.Endoh, J. Mater. Res. 182633 (2003)

7 M.Tamai, M.Nakamura, T.Isshiki, K.Nishio, H.Endoh and A.Nakahira., J. Mater. Sci: Mater in Med., 14617 (2003)

8 E.A.Stern Phys.Rev B 10 3027-3037 ((1974)

9 P.Lee and J.B.Pendry, Phys.Rev B. 112795 (1975)

10 J.E.Harries,D.W.L.Hukins and S.S.Hasnain, J.Phys.C 196859 (1986)

11 J.E.Harries, S.S.Hasnain and J.S.Shah, Calcif Tiss.Int 41346 (1987)

${ }^{12}$ K. Sakamoto, M. Okazaki, A. Nakahira and S. Yamaguchi, Bioceramics. 10241 (1997)

${ }^{13}$ Raoux, D.; Petiau, J.; Bondot, P.; Calao, G.; Fontaine, A,; Lagarde, P.; Levitz, P.; Loupias, G.; Sadoc, A. Reu. Roum. Phys., 151079 (1980)

${ }^{14}$ Lee, P. A.; Citrin, P. H.; Eisenberger, P.; Kincaid, B. M. R. Mod. Phys., 53769 (1981)

${ }^{15}$ Teo, B. K. Exafs: Basic Principles and Data Analysis; Springer: Berlin, (1986).

16 T.Minaki, T.Shiono, H.Aritani, T.Nishida, Soc.Mater.Sci.Jpn (ZAIRYO) 491211 (2000) 